How to reference this article

Balducci, M. A. (2021). Virgilio, le streghe e la sposa di San Francesco nella Divina Commedia. Italica Wratislaviensia, 12(2), 131-147.

DOI: http://dx.doi.org/10.15804/IW.2021.12.2.07

Marino Alberto Balducci

Carla Rossi Academy / International Institute of Italian Studies in Tuscany, Italia mab05@cra.phoenixfound.it

ORCID: 0000-0001-7407-6081

\title{
VIRGILIO, LE STREGHE E LA SPOSA DI SAN FRANCESCO NELLA DIVINA COMMEDIA
}

\section{VIRGIL, THE WITCHES, AND THE WIFE OF ST. FRANCIS IN THE DIVINE COMEDY}

\begin{abstract}
Through the hermeneutic-symbolic study of the Dantean figures of Virgil, the witches Manto and Erichto, and the mysterious woman loved by St. Francis, we reflect on the sense of magic and thaumaturgy in the Divine Comedy, presenting a new theological interpretation of the secret doctrine of Inferno IX. Virgil is shown in Dante's poem as the great Roman poet and as the magician of the Neapolitan folkloric traditions of the Middle Ages; he is celebrated but also ridiculed for his limitations. The Christian world, in fact, transcends the pagan idea of magic being Nature's proud minister, to advance a more complex thaumaturgic concept that indicates a miraculous event belonging not only to man but to the divine that is fused into the human dimension. Along these lines, the most essential characteristics and differences of Virgil the Magician's two infernal journeys in the Divine Comedy are analysed in detail: first, the one that is determined by the spells of Erichto, Lucan's witch, and which is therefore the work of ancient pagan black magic; second, the one that is Christianly predisposed by angelic inspiration, that is, by Beatrice, who is the symbol of a newer "celestial magic," which is, in turn, a special thaumaturgic power indicated by the paradisiac episode of St. Francis and his wife.
\end{abstract}

Keywords: Magic, Divine Comedy, Manto, Erichto, Saint Francis 
Secondo un metodo ermeneutico-simbolico, si riflette in questo studio sul senso del magico nel poema dantesco, proponendo una nuova interpretazione teologica della segreta dottrina a cui si allude nel canto IX infernale.

Per Dante - che è uomo del Medioevo - Virgilio è un grande poeta, e inoltre è anche un profeta che ha vaticinato l'avvento del Cristo nella famosa Ecloga Quarta. E poi Virgilio, grazie agli influssi delle leggende di origine napoletana, probabilmente legate anche all'errata attribuzione al poeta augusteo degli scritti magico-alchemici di un misterioso studioso cordobese, forse islamico ${ }^{1}$, secondo il nostro punto di vista ermeneutico era senz'altro da Dante (e non solo dalla sua epoca) considerato anche un mago. Questo, del resto, non ci dovrebbe stupire, per molte ragioni: difatti, nell'Età Antica, nel Medioevo e nel Rinascimento, rispetto alla prospettiva moderna post-leonardesca, era molto più ampia, sfumata e spiritualizzata la percezione globale di ciò che rappresentasse la scienza. Scienza in quei tempi era appunto un'idea che abbracciava le matematiche e razionali investigazioni della natura, ma anche lo studio dei più profondi segreti intuiti per vie misteriose e non razionali, pertanto vaticinati più che esperimentati e comprovati lucidamente in maniera inoppugnabile. In questo senso, non è casuale quell'uso dell'epiteto "saggio" che è posto al verso 89 , in apertura del primo canto della Divina Commedia, usato per il poeta di Roma, ad indicare una

${ }^{1}$ Cf. Lamendola, 2010. Su questo tema, si veda anche il classico studio di Spargo, 1934.

2 Per l'epiteto virgiliano ("saggio") e per Sapia, si ricordi quanto precisa Cicerone (De div. I, 31), cioè che in latino la parola saga si può riferire a una donna dotata di poteri speciali. Infatti, con sagae si indicavano donne sagge, cioè esperte conoscitrici dei lati occulti della realtà, capaci di forte intuizione, sagaci. Quest'ultimo aggettivo italiano si lega a questo contesto: deriva appunto da sagax, il quale a sua volta è connesso al verbo sagire nel senso di scandagliare, avere un fiuto particolare, intuire. Nel mondo antico e poi anche dopo, fino al Rinascimento, l'idea della saggezza include l'ambito astrologico, magico e spesso anche stregonesco. In questo senso è significativo anche un riferimento al nome di Sagana, la collega della strega Canidia nella Satira VIII di Orazio. Anche Stazio è definito come un saggio da Dante (cf. Purg. XXVII, 69): del resto egli stesso è emulo di Virgilio e pure cantore di magici riti infernali, quelli di Manto e di Teti (cf. Theb. IV, 463-585; X, 724-725; Ach. I, 135-140). 
profonda e misteriosa cultura caratteristica di un personaggio capace di investigare gli aspetti e gli scopi di questa nostra esistenza, dai gradi più umili, quelli dell'uomo pastore o agricoltore, nelle Bucoliche e nelle Georgiche, fino ai livelli dell'arte politica e militare e assieme del sacerdozio, nel grande poema imperiale (con il personaggio di Aeneas pater, dux et sacerdos $)^{3}$. Entro l'Eneide difatti, in quel famoso libro centrale che è il sesto, Virgilio svela anche molti segreti dell'oltretomba legati al pitagorismo e alle prospettive dell'alta filosofia del suo mondo grecolatino, sintetizzate da lui in immagini dense e suggestive. Egli è il nuovo Omero latino che vede la vita dell'uomo profondamente, anche nel buio, dentro i misteri oltremondani, e svolge investigazioni ben più complesse e articolate, nei riti e nelle visioni, rispetto al libro lambda dell'Odissea dove si narra la nèkyia, l'esplorazione del protagonista che è Ulisse nel regno dei morti. Virgilio è certo per Dante il modello assoluto dell'arte poetica in generale e, in particolare, per un'idea della morte come paidèia, come esperienza istruttiva, educativa. Virgilio è inoltre nella Divina Commedia pure un riflesso del negromante delle leggende napoletane, un incantatore che è in grado di dialogare e comandare i demòni dentro l'inferno. Se nella vita, secondo le varie storie partenopee, questo sapiente era capace di far compiere imprese impossibili ai suoi alleati infernali, come l'escavo di quella grotta famosa, la Crypta ${ }^{4}$, nell'altra vita, ormai diventato uno spettro, questo poeta è fatto oggetto di scherno e ingannato da vari demòni.

A ben vedere, per tutto l'inferno dantesco, Virgilio riesce a comandare o comunque a dominare parecchi demòni/mostri che fanno parte culturalmente di quel suo mondo dell'Antichità, in senso greco-latino

3 Pure le Bucoliche e le Georgiche cantano il "fare" creativo (e dunque, in un certo senso, "magico") dell'uomo, la naturale attitudine a trasformare i processi della natura per determinare cose più alte rispetto ai risultati normali del mondo animale e vegetale. Questo ad esempio è evidente nell'Ecloga III (80-83; 98-99), con i consigli sulle coltivazioni e l'allevamento del gregge, e nei carmi georgici in generale. Per la magia propriamente detta, giova ricordare la scena famosa della fattura d'amore nell'Ecloga VIII (62-109) e la descrizione dell'ippomane che è utile ai sortilegi di streghe nella Georgica III (280-283).

44 cf. D’Ovidio, 2012. 
e giudaico: basti pensare al suo incontro con il nocchiero Caronte, Minosse giudice, Cerbero, Pluto, il Minotauro e i Centauri, Gerione, i Giganti e, in fondo, anche Lucifero. Non si può dire lo stesso invece per i suoi scambi coi diavoli, presso le porte di Dite e in Malebolge: in questi casi, Virgilio non riesce a imporsi (cf. Inf. VIII, 82-130; IX, 1-15; XXIII, 140-148). Lui è umiliato piuttosto, è preso in giro.

Il degradamento nel comico della emblematica nobiltà virgiliana è molto importante all'interno della Divina Commedia. Virgilio infatti in quanto antico e pagano - è per sua natura storica e culturale un uomo ingannato, un uomo umiliato ${ }^{5}$. È così infatti che lui si presenta fin dalle prima parole (cf. Inf. I, 70-72).

Gli dèi pagani di quel suo tempo remoto sono bugiardi, la metafisica di quell'età che riusciva ad avere con Roma il dominio del mondo, nella politica della gran pace augustea, è difatti una menzogna, secondo il punto di vista cristiano. Roma è crollata alla fine, con i suoi dèi menzogneri e dunque demòni, come ci indica Sant'Agostino (cf. De civitate Dei I, 1-7). Generalmente parlando, i filosofi greci e latini, capitanati da Platone e da Aristotele, hanno esaltato un concetto divino come suprema ragione, autosufficienza ed equilibrio. Su di esso poi hanno certo in molti modi pensato, creando il concetto di quanto secondo loro è più grande nell'uomo, in base a un punto di vista che è razionale in senso umano e funzionale al successo dell'individuo nel mondo, attraverso il sublime esercizio della virtù che è mesòtes. Questa è un temperato equilibrio di forze contrapposte e, in fondo, una vera sintesi magica nel senso antico del termine; ma per il tempo cristiano - come ci dice San Paolo - quello che agli uomini del paganesimo sembra più lucida e chiara ragione è dunque inganno, è solo follia. E questo appunto secondo il Nuovo Dio: un Dio misterioso che fonda proprio su un piano che è irrazionale (la croce e l'uccisione di sé nel suo Figlio) il segreto di quella sua Onnipotenza Amorosa (cf. I Cor. III, 18-20; Rm. I, 21-22).

Pertanto, il demoniaco, che nell'inferno, come si è detto, schernisce direttamente o simbolicamente l'antico mago Virgilio, ci rappresenta un

5 Sull'incontro simbolico fra Dante e Virgilio nel Poema, si veda almeno Policastro, 2004. 
emblema della sconfitta inevitabile del mondo antico e del suo culto razionalistico olimpico, un culto capace di controllare le forze istintuali, ma mai di distruggerle o convertirle. Infatti, il tempo di conversione che esprime "Forza", di una più forte "magia" rispetto all'antica, è quello cristiano: questo è il momento del vero miracolo "folle", eppure capace di ricondurre le cose a una più alta ragione attraverso la sua follia, cioè la sua fede transrazionale, come ricorda San Paolo (cf. I Cor. III, 19).

Fondamentale per un esame ravvicinato di questo passaggio nella Divina Commedia dal primo antico concetto della magia a quello nuovo, cristiano ${ }^{6}$, è proprio il tempo del transito del pellegrino attraverso le porte di Dite per consumare l'ingresso nel cuore del cosiddetto male assoluto (peccato mortale) del centro e dell'area bassa d'inferno. Non solo Virgilio qui è umiliato dai molti demoni, ma anche le Erinni ora provano a fargli sentire la radicale impotenza a procedere (cf. Inf. IX, 34-39, apparse "ver l'alta torre" presso l'ingresso della città infernale (Inf. IX, 36).

La situazione ci sembra qui riecheggiare in tono di parodia dolorosa una specifica fra le svariate leggende del Medioevo napoletano intorno al Virgilio mago: quella delle bizzarre teste marmoree vaticinanti di Porta Nolana, disposte magicamente dal negromante poeta durante la vita nei luoghi partenopei ${ }^{7}$. E nell'inferno dantesco il gran savio latino non è certo più incantatore di statue metalliche (cf. Gervasio di Tilbury, 2009, pp. 61-63), ma invero è spaventato dai grandi poteri del meduseo che può trasmutare gli esseri umani nel marmo, lui ha paura di quelle Erinni che invocano la loro quarta sorella, la Gorgone (cf. Dell'Aquila, 1989).

6 Il concetto di "magia cristiana" sarà ampiamente chiarito nel Quattrocento. Cf. Pico della Mirandola, De hominis dignitate, § 37, 228-254.

7 Cf. Comparetti, 1941. L'episodio delle teste vaticinanti è narrato negli Otia imperialia di Gervasio di Tilbury (cf. Gervasio di Tilbury, 2009). Questo volume, pubblicato intorno al 1211, dedica largo spazio alle arti magiche di Virgilio e ai suoi prodigi, spesso operati grazie ai demòni, le forze basse della materia che lui sapeva gestire con la sua alta sapienza e competenza di ars mathematica, cioè di astrologia. L'influenza di Gervasio sulla Divina Commedia è stata studiata ad esempio da Paolo Cherchi (cf. 1989), della University of Chicago, per la descrizione del purgatorio come un luogo non sotterraneo, o da Claudia Di Fonzo (cf. 1999) che si è dedicata principalmente al Tilburiense come fonte nella prima cantica. 
Come si vede, la magia nera, quella fondata sopra l'orrore, lo sporco, sul vizio e il crimine e sul dolore, quella basata sull'abominio, può risultare alla fine incontrollabile da un mago sapiente che effonde il suo potere attraverso il controllo dell'energia aristotelica della mesòtes (cf. Etica Nicomachea, V), purificando i suoi impulsi bestiali. Così, nella coscienza dell'uomo, il "femminino" nei suoi aspetti più negativi (cioè la furiosa passione istintuale) è destinato alla fine a prevalere sul polo "maschile" della giustizia razionalmente ordinata: questo rivela a noi Dante attraverso i suoi simboli. Infatti, come si è visto, Virgilio nella Divina Commedia viene osteggiato dalle Erinni e deriso dai diavoli. Inoltre, lui è pure succube delle maliarde più nere, di Manto e di Eritone.

Nel primo caso, la strega tebana, la figlia del vate Tiresia, appare in Malebolge nell'area della magia fraudolenta (cf. Inf. XX, 82-99) ${ }^{8}$. Qui il maestro di Dante ricorda i propri natali, la propria terra di origine; e dunque adesso corregge come un errore quanto ci aveva descritto nei versi del suo capolavoro (cf. Aen. X, 198-203). Mantova entro il poema di Roma appariva come fondata dal figlio di quella strega nefasta e del fiume d'Etruria, da Ocno cioè, che ereditava dal padre i poteri della famosa terra dei savi etruschi conoscitori del cielo. Mantova e dunque Virgilio (col suo messaggio poetico profetizzante il destino immortale di Roma e dell'Impero Romano) sembravano allora simbolicamente legati alla potenza della "ragione maschile" e quindi alla forza della magia superiore connessa all'astrologia. Nella Divina Commedia al contrario la guida dantesca confessa ciò che ha scoperto al di là della vita, nell'oltretomba: nella coscienza di Ocno (così come dentro la psiche di ogni mortale), la forza più forte - più forte di tutte le stelle e di ogni ragione deriva dal fondo abissale e tremendo di sangue e di ingiustizia, di orrori: il fondo di Manto, la madre, la strega.

Come si afferma in quel ventesimo canto infernale, Mantova è stata scelta perché paludosa, una terra di putrefazione (cf. Aen. X, 198-203); e la veggente sapeva che solo da questa immondizia (in un senso lato, che è fisico e anche morale) è dunque possibile trarre il potere più gran-

${ }^{8}$ Intorno alla bolgia dei maghi e indovini, si vedano: Bàrberi Squarotti, 1972; Boitani, 2013; Gareffi, 2007; Hollander, 1980; Paratore, 1979-1980. 
de nel mondo storico-materiale che è magia nera. Così Virgilio simbolicamente, entro il poema di Dante, si mostra succube della tebana, ne è influenzato fin dalle origini perché ora appieno si rende conto che è nato da quella terra di stregonerie, sopra la tomba di quella maga. La sua sapienza e profezia e magia di gran savio son collegate nel fondo a quella donna e al suo potere malvagio che sempre può prevalere senza che lui riesca a dominarlo con la sua scienza. Questa visione si lega in Dante anche all'idea del peccato originario in senso precisamente giudaico-cristiano: l'uomo creato dal Padre Celeste ha preferito all'origine udire e seguire le indicazioni della serpe, così ha colto e mangiato il frutto proibito offerto da Eva (cioè la parte "femminile" istintuale e passionale della sua stessa natura). Come ricorda San Paolo, dentro di noi c'è sempre una forza molto più forte del razionale autocontrollo (cf. $R m$. VII, 18-23).

In questo senso, il caso di Erichto nella Divina Commedia è ancora più illuminante, e ci rivela altre cose. La donna è come Manto una terribile strega, figura fondamentale di un altro grande poema latino che è posteriore all'Eneide, cioè la Farsalia del cordobese Lucano. La negromante in questione è esperta di magia nera; non è immortale come gli dèi dell'Olimpo, ma nel suo tempo storico è davvero invincibile, domina gli uomini e pure gli dèi - in un modo che è orrendo e sconvolgente - con i suoi sporchi crimini ed il potere dell'odio (cf. Phars. VI, 523-532). I suoi scongiuri sembrano onnipotenti; e così dunque gli dèi impauriti concedono tutto all'orrenda maliarda, temono quella sua voce terribile e poi soprattutto l'evocazione del mostro innominabile: il Demogorgòne (cf. Phars. VI, 527-528). E ora Virgilio rivela a Dante che proprio lui, dopo la morte, è stato asservito da quella tessala maga: fu "congiurato" da lei che strappa le anime sotto la terra dal nero dell'indistinto (cf. Inf. IX, 22-27).

Ver è ch'altra fỉata qua giù fui, congiurato da quella Eritón cruda che richiamava l'ombre a' corpi sui.

Di poco era di me la carne nuda, ch'ella mi fece intrar dentr'a quel muro, per trarne un spirto del cerchio di Giuda. (Inf. IX, 22-27) 
Erìchto, nella visione di Dante, ha scelto Virgilio come ideale strumento maligno, e questo certo per la sapienza e magia del grande poeta, per i suoi poteri. Erichto lo ha evocato e asservito, portandolo dunque ad agire nell'ombra ctonia proprio secondo le leggi blasfeme del crimine che lei controlla, nell'infrazione totale di quei principi di integrità e di equilibrio rappresentati dal dignitoso contesto (il castello infernale) che il gran poeta romano aveva raggiunto nell' aldilà, come se fosse il suo premio spirituale. Così la fortezza del bello e dell'onore viene espugnata. E questo è ironico, certo. Il vento che trema in quella selva del limbo (cf. Inf. IV, 27, 150), pare ghiacciare la superficie del fiumicello, che sembra un fossato difensivo, mentre si può oltrepassare come se fosse di terra, di "terra dura" (Inf. IV, 109) ${ }^{10}$. L'autocontrollo della mesòtes, il razionalismo senza passione, glaciale (apàtheia), considerato suprema virtù da Aristotele e dagli antichi filosofi classici, sembra ad Agostino cristiano immanitas animi, solo un'orrenda mostruosità (cf. De civitate Dei, XIV, 9, 4).

Questa freddezza non certo nobilita l'uomo in senso cristiano, perché impedisce al nostro cuore di sciogliersi e di commuoversi quando si trova davanti al dolore dei propri simili. Dunque, tale freddezza rischia alla fine di degradare l'umano e consegnarlo al piano feroce, diabolico. Così Virgilio, che storicamente non era umile, perché guidato dalla fiducia "maschile" nella ragione e nel freddo autocontrollo dell'io onnipotente, nella Divina Commedia ironicamente viene costretto, da quella strega che espugna il suo castello con la passione malvagia, a degradarsi immoralmente sempre più giù, fino $\mathrm{al}$ fondo più basso e più sporco di tutto l'inferno. Infine, lui è guida di un traditore del cerchio di Giuda, fantasma che si è macchiato di un crimine orrendo ma, proprio per questo, poiché è conficcato nel basamento della voragine nera, conosce bene tutto l'inferno che ha attraversato, e poi possiede il potere più forte delle energie della terra settentrionale: energie peccaminose utili al fine di ana-

9 Il virgiliano viaggio maligno voluto da Erìchto è avvenuto dopo la morte del poeta (19 a.C), comunque prima della discesa di Cristo agli inferi (33 d.C) secondo quanto indicato in Inf. XII, 34-39.

10 La nostra interpretazione risente anche del significato del tipico simbolo dantesco dell'acqua pietrificata (cioè di ghiaccio) associata al disamore: cf. Rime, CII, 25-36. 
lizzare e prevedere precisamente gli eventi futuri, per favorire avidità di progetti materialistici. Inoltre, Erichto ha costretto il famoso spettro romano, facendo appello alla bestia peccaminosa all'interno di lui come di ogni mortale (rappresentata emblematicamente in questo caso da Manto, la madre), una bestia che si mostra tenuta a bada e celata, ma mai distrutta dall'autocontrollo e dalla sapienza dell'uomo poeta. Lui, che credeva di dominare e asservire il demoniaco a tutti i suoi scopi nel mondo (come nel caso di quella Crypta famosa a Posillipo, fatta scavare da vari demòni soltanto in una notte: cf. Lamendola, 2010), finisce quindi per diventare un famiglio delle arti più nere. La situazione è paradossale e tragicomica, anche perché ci ricorda altri eventi famosi delle leggende napoletane sul mago Virgilio, in particolare connesse alle sue magiche teste di morti parlanti, capaci di rivelare il futuro e da lui controllate (cf. Comparetti, 1941); ma ora, in questo particolare episodio dantesco, Virgilio è un servo di necromanzia e certo non è il necromante.

Questa vicenda, narrata nel canto IX infernale, sconcerta ed è ricchissima di implicazioni ermeneutiche. Durante il primo viaggio notturno di cui Virgilio ci dice - quello voluto da Erichto - egli era riuscito a comandare i demoni con la sua scienza di alta magia razionale e la potenza più oscura della padrona selvaggia, entrando senza problemi nel cuore di tutto l'inferno; ma è interessante che poi, una volta con Dante e nel tempo cristiano, lui debba ammettere la sua sconfitta, perché non riesce a farsi aprire da solo le porte di Dite.

Il tema della sconfitta nel mondo giudaico-cristiano si associa al peccare dell'uomo che indebolisce. Comunque, secondo il punto di vista dantesco, esiste un bene nascosto dentro la selva che rappresenta il nostro peccato (cf. Inf. I, 8). Per un cristiano è unico l'Essere, esiste un solo assoluto: l'Amore, la cui essenza esclusiva è rappresentata dal Bene. Dunque, la selva peccaminosa non è solo male, non è solamente il luogo della sconfitta. In essa, può anche avvenire l'incontro fondamentale per la salvezza dell'individuo smarrito: e questo per Dante è l'incontro con il poeta Virgilio, inviato e istruito dall'anima bella di Beatrice, colei che è scesa dentro l'inferno ${ }^{11}$.

11 Per l'incontro spirituale tra Virgilio e Beatrice, si possono vedere Aversano, 1992; Basile, 2004; Fallani, 1986; Masciandaro, 1972; M. Picone, 2008. 
Beatrice, che a pieno diritto è anima del paradiso, si mostra dunque nel regno infernale senza paura di quel suo "incendio" diabolico (Inf. II, 93.); rivela così, anche nel buio, il permanere immortale del bello e dell'amore, valori inestinguibili fra i vivi e i morti. La Gentilissima mostra la Grazia, il dono della salvezza che è immerso segretamente in ognuno, anche dentro la morte, il peccato e l'inferno, estremi ignobili e sporchi, orrori indegni che paiono proprio non legittimarlo e non meritarlo. Questo è il messaggio evangelico più sconvolgente, quello del prologo giovanneo, che è tramandato dal testo greco originario seguito a Bisanzio, nel mondo spirituale di Giustiniano glorificato da Dante (cf. Par. VI, 10-33):

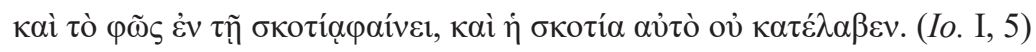

La Luce è scesa dentro le tenebre, dentro l'inferno; e qui le tenebre non l'hanno vinta ${ }^{12}$. Così l'inferno - con tutto il male e l'errore, con tutte le stregonerie dei demoni e di Erichto - non può far nulla contro una Forza diversa da tutte le forze esteriori della materia. Nella bellezza Beatrice riflette nella sua carne una simile Forza: essa è il quinto elemento eternale che è spirito e quintessenza. Questo elemento fa parte della materia, ma non è concreto, tangibile: è immateriale ed immortale, a differenza degli altri quattro elementi. L'uomo lo rispecchia solo negando la sua orgogliosa autosufficienza, lasciandosi attraversare da una Potenza al di sopra di tutti i confini dell'io.

Il male che serpeggia fra i nostri istinti nefasti della coscienza ha pure un bene occultato, di certo, perché, come dice San Paolo, esso ha un potere che è buono (e ne è inconsapevole): abbassa il nostro orgoglio, ci umilia, ci fa avvertire inferiori alle circostanze, ci abbatte su quella terra che ci ha generati e ci fa sentire che ad essa apparteniamo ogni

12 Anche nel nostro occidente cristiano romano, dal 2008, per questo versetto giovanneo si segue il senso del testo greco originario, approvato e tradotto dalla Conferenza Episcopale Italiana. 
volta - al di là del nostro orgoglio demente - e che a quella, morendo, comunemente dobbiamo tornare ${ }^{13}$.

Allora annullandoci, annichilendoci in umiliazione, riconoscendo che solo le nostre forze (le forze capitanate dall'io razionale) sono incapaci di favorire la nostra salvezza, possiamo aprirci all'ascolto dell'Altro che è altro-da-noi: così fa Dante nella sua selva, mentre lui perde ogni "speranza dell'altezza", si abbandona a Virgilio il quale, a sua volta, si è abbandonato a Beatrice che ora parla nella sua mente (Inf. I, 54). La bella poesia di Virgilio adorata da Dante inizia al dialogo e, ugualmente, il bello umano e sovrumano di Beatrice convince il poeta romano intristito dentro il suo inferno.

Proprio l'humilitas è il mistero centrale ristrutturante del Cristianesimo: in esso il soggetto umiliato e umile può incontrarsi con un'idea del divino che è essa stessa umiltà, in quanto esprime il concetto cristiano di un Dio che ha tutto, ma non accetta che l'uomo sia infelice a causa delle sue libere scelte. Dunque, il Divino misericordioso e innamorato dell'uomo discende sul piano umano col Figlio, che prova a strappare gli umani al dolore: ma non costringe davvero, in nessun modo. Così Beatrice, nella Divina Commedia, diviene perfettamente imago Christi anche e soprattutto nel senso che lei rappresenta ovunque - pure all'inferno e soprattutto all'inferno - una possibile fuga. La sua bellezza quintessenziale ha la virtù di persuadere Virgilio, senza obbligarlo. Beatrice evoca spettri dei morti per liberarli attraverso una preghiera d'Amore, come Gregorio, l'antico Papa, che liberava il pagano Traiano dalla prigione infernale (cf. Purg. X, 73-93; Par. XX, 43-48, 103-117). Lei non "congiura", cioè non costringe, come fa Erichto con i demoni e con Virgilio (cf. Inf. IX, 22-30), anch'esso demonio, cioè sviato da demoniaci egoisti e materialisti pensieri, in quanto pagano ${ }^{14}$. Beatrice si mostra e naturalmente (con il potere del Bello) suscita una risposta nel morto poeta di Roma sensibile alle armonie. Lo spettro romano così, dalla for-

13 "et ex magnitudine revelationum. Propter quod, ne extollar, datus est mihi stimulus carni, angelus Satanae, ut me colaphizet, ne extoller" (II Cor., XII, 7).

14 Per Dante infatti gli angeli sono del tutto analoghi alle idee platoniche: pensieri puri, perfetti, emanazioni del Sommo Bene (cf. Convivio, II, 4). I demòni saranno allora allusioni ai pensieri sbagliati, menzogne mentali. 
za della Gentilissima, è incoraggiato a parlare ed a chiedere a quella donna - liberamente, si badi bene - di farlo agire per lei. E Dante uomo, che ci rappresenta, è proprio salvato dalla bellezza: non solamente dalla bellezza del mondo creato (l'aspetto meraviglioso dell'alba rincuora infatti il pellegrino smarrito, ma non lo scampa dalle tre fiere: cf. Inf. I, 13-60), piuttosto dal bello della natura filtrato dalla sapienza dell'arte e, dunque, da Beatrice... dentro Virgilio. Siamo al cospetto di un umile amore divino innamorato dell'uomo che, pur di salvarlo, accetta di comunicare al suo stesso livello concreto e naturale. Il potere catartico di questo amore umiliato nell'ombra sembra insignificante, inesistente, ma ha un'infinita potenza che si può chiamare a buon diritto "magia" ("magia cristiana" o come si dice più comunemente "taumaturgia"), se con questo termine noi vogliamo indicare un prodigioso potere di trasformazione (cf. Coleston, 2007). Ed una tale "magia" a nostro avviso è il segreto nascosto "sotto "1 velame", in quello che è certo un luogo del testo della Divina Commedia dei più dibattuti criticamente (Inf. IX, 63; cf. Dell'Aquila, 1989).

O voi ch'avete li 'ntelletti sani, mirate la dottrina che s'asconde sotto 'l velame de li versi strani.

$$
\text { Inf. IX, 61-63 }
$$

Come si è visto, nel canto IX infernale Virgilio è a un tratto sconfitto da tutti i demoni là a Dite, che gli rifiutano il transito oltre le porte. Virgilio, nella veggenza, avverte a questo punto che un messo celeste potrà favorire il passaggio; ma questi non viene, ritarda, come si è visto fare a Beatrice, la quale giunge in aiuto di Dante quando lui è già smarrito dentro la selva (cf. Inf. II, 64-66). Il duce latino non se ne accorge, ma pure - davanti a Dite e alle Erinni, le quali compiono l'evocazione del mostro che è orrore e angoscia di morte (Medusa) - è proprio lui a favorire l'arrivo del salvatore dal cielo, il messo... la Grazia. E il maestro Virgilio lo fa nel preciso momento in cui si abbandona all'Amore che è Forza: sì, forte più della morte e dell'odio del meduseo, che è magia nera. La magia nuova - cristiana - nasce difatti da un 'vuoto', cioè a dire da una totale assenza di orgoglio intellettuale. Virgilio, a questo punto, 
non prova più a risolvere il suo problema di quel passaggio negato oltre le porte, col ragionamento del suo pensiero finito e con il dialogare con gli ufficiali maligni che sono i demoni. Al contrario, lui si abbandona. È abbandonato all'amore - Amore Infinito - il poeta latino, come una donna, come una madre o un'amante irrazionale. Lui è uno spettro senza materia tangibile, eppure non si accontenta di fare voltare indietro il suo protetto e poi di fargli coprire gli occhi con le sue mani: no, per davvero. Lui su quegli occhi vuol mettere anche le proprie palme senz'altro, quelle sue palme inconsistenti e inutili razionalmente, comunque piene di un amoroso sentire che supera ogni ragione, ogni umana ragione e che attira così... la potenza dell'Infinito (cf. Inf. IX, 52-63). Lui nell'amore mostra fiducia nell'ombra intangibile, come farà anche Stazio, nel purgatorio, dimenticando che il suo maestro Virgilio è un fantasma (cf. Purg. XXI, 130-136).

Ecco il miracolo umile e immenso che brilla "sotto il velame" (Inf. IX, 63). Virgilio, sconfitto e umiliato nella sua scienza e magia, attira come un magnete l'evento meraviglioso (l'apparizione dell'angelo) senza saperlo. Eccola quella sfuggente "dottrina" nascosta che è l'unica per i cristiani, del resto, e risolutiva: 1'Amore, amore per l'uomo concreto, di carne, e amore per Dio che non si vede e non possiamo toccare. Senza alcun dubbio è proprio l'humilitas che rappresenta l'aspetto centrale del Cristianesimo: essa è uno stato di svuotamento (kénosis) che predispone a ricevere ogni virtù razionale e spirituale. Ed è una morte pertanto, la "sora nostra Morte" che è valorizzata e cantata da San Francesco (Laudes creaturarum, 27).

Il Santo di Assisi è una figura fondamentale per Dante che poeticamente approfondisce il rapporto tra francescanesimo, morte e povertà, nel canto XI del Paradiso ${ }^{15}$. La "donna" (Par. XI, 58) amata dal Frate, a cui il poeta si riferisce in senso metaforico ${ }^{16}$, si identifica precisamente col culto francescano della umiltà/humilitas come povertà/paupertas

15 Per un'osservazione critica generale di quest'episodio paradisiaco, si veda Distante, 1986.

${ }^{16}$ Sul tema del folle innamoramento di San Francesco nel Paradiso cf. Allworthy Armstrong, 1973; Chiarenza, 1993; Deen Schildgen, 2000; Herzman,1982; Mineo, 1992. 
(cf. Par. XI, 74-75): questa è una 'donna-porta' (cf. ibid., 50), una donna che sembra racchiudere nella sua attraente intimità un concetto diverso dell'erotismo e del piacere.

Non era ancor molto lontan da l'orto,

ch'el cominciò a far sentir la terra

de la sua gran virtute alcun conforto;

ché per tal donna, giovinetto, in guerra

del padre corse, a cui, come a la morte,

la porta del piacer nessun diserra; (XI, 55-60) (Par. XI, 55-60)

Notiamo ora che questa donna, sconvolgendo i sensi e il pensiero del Santo in amore, sia indicata dal poeta come "feroce" (Par. XI, 70): cioè ferox, o per meglio dire fera, e dunque selvaggia, bestiale ${ }^{17}$. Lei rappresenta l'impatto della follia nella mente. È una rottura di schemi, è il furore: si abbatte sulle categorie dell'essere, distrugge quindi le sicurezze di ogni spirito geometrico. Feroce allora è la donna di frate Francesco, così come "crude" (cf. Inf. IX, 23; XX, 82) e crudeli vengono definite dal Poeta le orribili signore dei malefici notturni di cui si è detto, cioè Manto e la perfida Erichto ${ }^{18}$ profanatrice di tombe e di cadaveri. Sul piano semantico siamo di fronte ad uno stesso furore, ad una stessa distruzione degli schematismi del pensiero; ma il segno dell'energia che si esprime nell'impeto e amore di San Francesco è senza dubbio diverso $^{19}$. Le antiche maghe sono animate da odio rispetto all'unità/armonia divina; così quel furor rappresenta per loro una porta di "deiezione", per così dire, e dunque è esilio da un armonioso rapporto d'essere naturale originario e inizio, dunque, improrogabile di una guerra fra i vari opposti, una guerra all'insegna dell'egoismo e del dualismo. Invece,

17 Preferiamo cogliere una specifica indicazione brutale nel termine dantesco, un'indicazione che ci riporta al senso acquisito da quest'ultimo nel canto XIII dell'Inferno (94: "Quando si parte l'anima feroce") secondo la lettura del Buti, 1858-1862: "imperò che [il suicida] come fiera incrudelisce contro se medesimo".

18 Entrambe, infatti, sono descritte come "crude": cf. Inf. IX 23; XX 82.

19 Su questa medesima linea interpretativa, Erich Auerbach (1984, p. 231) metteva in luce l'aspetto sorprendente - ripugnante, crudele e insieme sublime - di tale unione fra il Santo e Paupertas. 
la francescana paupertas rinnega il senso di divisione, abiura quindi in uno slancio la terra d'esilio, nel giubilo muto di un'estasi mistica tutta animata dal fuoco d'amore che, proprio entro il dolore della rottura del limite razionale egoico e poi oltre questo, ristabilisce unità, distruggendo così - nella mente e nel corpo - ogni catena di differenze.

L'amore per la nox animi, cioè l'amore per questa donna "scura" (Par. XI, 65), determina dunque radicali trasformazioni sul piano sentimentale (amore degli altri $>$ rinnegamento dell'egoismo e, quindi del senso di proprietà) e su quello intellettuale (amore dell'Altro che è mistero trascendentale $>$ superamento del logico raziocinio e costituirsi di un armonioso pensiero, in cui ogni senso di differenza si perde). Il bello e il brutto, il dolce e l'amaro: tutto è riunito, trasceso in quella tenebra francescana... luminosissima. La donna nera così, necessitate cogente, si associa in amorosi connubi a Francesco, al sole dell'India, all'Oriente e assieme ad Assisi che indica l'Occidente: porta la notte e l'oscurità della sua pelle orientale dentro la luce in Italia (cf. Par. XI, 49-66). Qui siamo oltre ogni logica umana e anche oltre le contraddizioni. Tutto è piacere - anche dentro il dolore - ed è "perfetta letizia" (Fioretti di San Francesco, VIII). È il matrimonio di ogni contrario, ed è pure la santa follia di un «dolce sguardo» che è pieno di "meraviglia" (Par. XI, 77), proprio perché ora è in grado di penetrare più oltre l'avvertimento dei contrasti fisici (percezione sensuale-razionale), per cogliere quindi nel mondo l'essenza di una "concordia" (Par. XI, 76) purissima che anima il cuore di tutte le creature (percezione serafica: cf. ibid., 76). Questa dolcezza di sentimento e visione fa germinare "pensieri santi" (Par. XI, 77), armoniosi, perché è senza ombre di dubbi, di antitesi e contraddizioni. Questo è amoroso pensare la Verità, senza più sforzo. Anche il furore più oscuro di quelle streghe dei tempi remoti - nell'umiltà di un ascolto profondo - riesce così a specchiare la Forza che, sola, ci fa risorgere e porta fino alle stelle. 


\section{BIBLIOGRAFIA}

Allworthy Armstrong, E. (1973). Saint Francis: Nature Mystic. The Derivation and Significance of the Nature Stories in the Franciscan Legend. Berkeley-CA: University of California Press.

Auerbach, E. (1984). Studi su Dante (translated by M.L. De Pieri Bonino, \& D. Della Terza). Milano: Feltrinelli.

Aversano, M. (1992). Note al canto II dell" "Inferno". In idem, Un nuovo Dante. Il realismo teologico dell'Inferno (pp. 55-56). Avellino: Il Calamaio.

Bàrberi Squarotti, G. (1972). Inferno XX. L'artificio dell'eternità (pp. 235-281). Verona: Fiorini.

Basile, B. (2004). O donna di virtù ("Inf." II, 76). Rivista di studi danteschi, $4 / 1,161-183$.

Boitani, P. (2013). Tiresias and the Soothsayers. In J.J. Kinder, \& D. Glenn (Eds.), Legato con amore in un volume: Essays in Honour of John A. Scott (pp. 205-219). Firenze: Olschki.

Cherchi, P. (1989). Gervase of Tilbury and the Birth of Purgatory. Medioevo Romanzo, 1/14, 97-110.

Chiarenza, M. (1993). Dante's Lady Poverty. Dante Studies, 3, 153-175.

Brown, C. (2007). Magical Christianity: The Power of Symbols for Spiritual Renewal. Wheaton - IL: Quest Books.

Comparetti, D. (1941). Virgilio nella tradizione letteraria fino a Dante (II, 9). In idem, Virgilio nel Medioevo. Firenze: La Nuova Italia.

D’Ovidio, S. (2012). Boccaccio, Virgilio e la Madonna di Piedigrotta. In G. Alfano, T. D’Urso, \& A. Perriccioli Saggese (Eds.), Boccaccio angioino. Materiali per la storia culturale di Napoli nel Trecento (pp. 329-346). Bruxelles: Peter Lang.

Di Fonzo, C. (1999). La diffrazione per istituto e la tradizione dell' 'Ottimo Commento': opus practicum del commentatore (Inferno XXVIII, 6-12). Il caso di Gervasio Tilliberense. Electronic Bulletin of the Dante Society of America, January. Retrieved from http://www.princeton. edu/\%7Edantelebdsa/index.html

Distante, C. (1986). Lettura dell'XI canto del Paradiso. Lingua e Letteratura, $15,23-33$.

Fallani, G. (1986). Canto II. In P. Giannantonio (Ed.), Lectura Dantis Neapolitana. Inferno (pp. 17-26). Napoli: Loffredo.

Gareffi, A. (1980). Qui vive la pietà quand'è ben morta: Inferno, XX. Sincronie, 21-22, 183-188. 
Gervasio di Tilbury (2009). Il libro delle meraviglie (edited by E. Bartoli). Pisa: Pacini.

Hollander, R. (2007). The Tragedy of Divination in Inferno XX. In idem, Studies in Dante (pp. 131-218). Ravenna: Longo.

Herzman, R. (1982). Dante and Francis. Franciscan Studies, 42, 96-114.

Lamendola, F. (2010). Il culto di Virgilio nel Medioevo. Retrieved from http:// www.centrostudilaruna.it/il-culto-di-virgilio-nel-medioevo.html.

Masciandaro, F. (1972). "Inferno" I-II: il dramma della conversione e il tempo. Studi danteschi, 49, 1-26.

Mineo, N. (1992). La "vita" di san Francesco nella "festa di paradiso". In A. Mellone O.F.M. (Ed.), Lectura Dantis Metelliana, 2 (pp. 223-320). Roma: Bulzoni.

Paratore, E. (1979-1980). Il canto XX dell'Inferno. Studi danteschi, 52, $149-169$.

Picone, M. (2008). "Inferno" I e II: l'uno e l'altro viaggio. In D. Cofano, \& S. Valerio (Eds.), Versi controversi. Letture dantesche (pp. 7-38). Foggia: Edizioni del Rosone.

Policastro, G. (2004). Appunti sulla catabasi di Dante nella ripresa di alcuni motivi di autori classici: il topos drammatico dell'incontro e il riuso simbolico del rito. Italianistica. Rivista di letteratura italiana, 33, 11-27.

Schildgen, B.D. (2000). Dante and the Orient. Champaign - IL: University of Illinois Press.

Spargo, J.W. (1934). Virgil the Necromancer. Cambridge - MA: Harvard University Press.

Riassunto: Attraverso uno studio ermeneutico-simbolico delle figure dantesche di Virgilio, delle streghe Manto ed Erichto e della sposa di San Francesco si riflette sul senso del magico e del taumaturgico nella Divina Commedia, proponendo una nuova interpretazione teologica della segreta "dottrina" a cui si allude nel canto IX infernale. Virgilio viene mostrato nel poema dantesco come il grande poeta latino e come il mago delle tradizioni folkloriche napoletane del Medioevo: così è celebrato ma anche ridicolizzato per i suoi limiti. Il mondo cristiano, infatti, trascende l'idea pagana della magia che è la signora orgogliosa della natura, per avanzare un "concetto magico" molto più vasto e complesso, evento non più soltanto dell'uomo ma del divino che è fuso e integrato nell'uomo. Su questa linea, si analizzano nei dettagli le più essenziali caratteristiche e differenze dei due viaggi infernali del mago Virgilio nella Divina Commedia: quello che è determinato dagli scongiuri di Erichto, la strega, ed è dunque opera di magia nera pagana antica, e quello che è predisposto cristianamente dall'ispiratrice angelicata, cioè da Beatrice che è simbolo della bellezza foriera di una "magia celestiale" più nuova, cioè una forma di taumaturgia indicata dalla figura paradisiaca di San Francesco e dalla sua sposa.

Parole chiave: Divina Commedia, Manto, Erichto, San Francesco, magia 